\title{
Basics of spectropolarimetry
}

\author{
John D. Landstreet \\ University of Western Ontario, London, Canada and \\ Armagh Observatory, Armagh, Northern Ireland \\ email: jlandstr@uwo.ca
}

\begin{abstract}
Many astronomical sources of radiation emit polarised radiation, for example because of the presence of a disk which produces linear polarisation by scattering some photospheric radiation, or because of the presence of a magnetic field, which leads to circular and sometimes linear polarisation of spectral line profiles. Measuring the wavelength dependence of the polarisation of radiation from such sources can reveal valuable and interesting constraints on the nature of the objects observed. This paper summarises the basic ideas of spectropolarimetry and describes some of the information it can provide.
\end{abstract}

Keywords. Polarisation, instrumentation: polarimeters, techniques: polarimetric, stars: magnetic fields, stars: circumstellar matter

\section{Introduction}

Normal stellar spectroscopy starts with measurement of the intensity of a beam of starlight as a function of wavelength. It is well known that such data provide an astonishing range of valuable information about the light source. However, spectroscopy does not exhaust the available information in the light beam. The light may be linearly and/or circularly polarised. Measurement of the polarisation state of the light as a function of wavelength, known as spectropolarimetry, can provide new and valuable constraints on the geometric structure of the light source (for example, on the shape and state of a circumstellar disk), or reveal the strength and structure of a magnetic field present in the star.

This paper is intended to provide a simple introduction to the subject of spectropolarimetry. I will qualitatively describe how polarisation of starlight can arise from the geometry of the source or a magnetic field. I will then discuss some basic methods of measuring the wavelength dependence of polarisation, and in particular explain how it is possible to reliably detect polarisation as low as one part in $10^{5}$. Finally I will discuss how important it is to be both careful and critical in measuring such tiny effects.

\section{Linear polarisation due to scattering}

Everyone is familiar with some of the phenomena of linear polarisation. If you look at sunlight reflected obliquely from a puddle of water through polarising sunglasses, the intensity of the light reflected by the puddle is diminished relative to the brightness of the surroundings. If you take off the polarising sunglasses and look through them as you rotate them around an axis normal to one lens, the brightness of the reflected light varies. The brightness is near its minimum value with the glasses in their normal orientation, and maximum when the glasses are at $90^{\circ}$ to this orientation.

Light is a transverse wave of electricity and magnetism, and if the direction of the electric vector is not distributed randomly around the direction of propagation, the light is said to be linearly polarised. The direction of the average electric vector is the plane of 


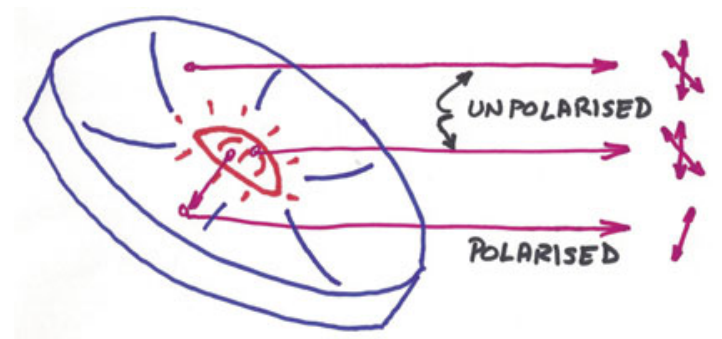

Figure 1. Polarisation of light from a star-disk system. The light emitted by both the stellar photosphere and by the hot circumstellar disk is essentially unpolarised, but the photospheric light that is scattered by free electrons in the disk is strongly polarised in the plane perpendicular to the plane containing the incident and scattered light beams.

polarisation, and the difference between the light intensity in that plane and the intensity in the orthogonal plane, divided by the total intensity, is the fractional polarisation.

In the puddle reflection observation described above, the reflection of light polarised parallel to the plane of reflection is less efficient than reflection of light polarised parallel to the surface of the puddle, so the reflected light has net polarisation parallel to the plane of the surface of the puddle.

This experiment illustrates the basic ideas of astronomical polarisation. Natural phenomena can preferentially emit, reflect, or scatter light with a preferred plane of plarisation. The polarisation of the light then carries information about the source or the reflection phenomenon. This information can be extracted by measuring the direction and amplitude of the polarisation. This is done by using a filter that passes one direction of polarisation but not the orthogonal direction (the sunglasses, whose lenses transmit vertically polarised light but not horizontally polarised light), and measuring the intensity of the transmitted light in each of the two orthogonal polarisation planes.

A typical astronomical situation leading to linearly polarised light is shown in Fig. 1. A star is surrounded by an accretion disk (a Herbig AeBe star, a mass transfer binary, etc.) or a decretion disk (a classical Be star, etc.) which is heated by the central star. The distant observer sees (1) light coming directly from the stellar photosphere, (2) light emitted thermally by the hot disk material, and (3) photospheric light that has been scattered by the free electrons in the disk. Components (1) and (2) are unpolarised, but component (3) is strongly linearly polarised because the scattering material in the disk is not distributed around the star in a spherically symmetric way.

Detection of linear polarisation from a star showing evidence of circumstellar material immediately allows one to conclude that the distribution of material around the star is not spherically symmetric, but is somehow flattened. This usually means that the matter is in a disk of some kind. Observing the spectrum of the linear polarisation of such a system can provide much further information about the relative importance of emission and scattering phenomena. For example, scattered (and thus polarised) photons may have to pass through part of the disk on their way to the observer, and so strong absorption in the disk can reduce the number of scattered photons escaping. Because of this, the wavelength dependence of polarisation can be used to constrain such model parameters as disk inclination to the line of sight, disk density, and disk thermal structure.

Some recent articles discussing the use of spectropolarimetry to understand the circumstellar material around hot stars include Vink et al. (2005); Davies et al. (2007); Carciofi et al. (2007); Halonen \& Jones (2013). 
There are some basic points to keep in mind about linear spectropolarimetry of stars.

- The observed fractional polarisation $p$ due to scattering is of the order of $p \sim$ (fraction of scattered photons). (geometric factor). Observation of non-zero linear polarisation, so that (geometric factor $) \neq 0$, reveals immediately that the system departs from spherical symmetry.

- Fractional polarisation values are often small in astrophysical situations. Circumstellar disks frequently lead to linear polarisation of order $1 \%$ or less. This implies that linear polarisation spectra usually require a lot of photons, of order $10^{6}-10^{10}$ photons per final (possibly binned or smoothed) pixel, in order to have measurement uncertainties that are small compared to the expected polarisation.

- The scattered photons usually require some detailed modelling in order to extract quantitatively useful information about a system.

\section{Zeeman splitting and polarisation in spectral lines due to magnetic fields}

One of the most widely exploited areas of spectropolarimetry is the use of (mostly circularly) polarised spectra to study magnetic fields in stars. This kind of measurement relies on polarisation produced by the Zeeman effect in spectral lines, which allows detection and (often) modelling of even very weak magnetic fields (down to the level of $\sim 1$ Gauss $\sim 10^{-4}$ Tesla).

The Zeeman effect arises from the fact that each energy level (state) of an atom has a magnetic moment, of the order of $e \hbar / 2 m c$, which is aligned with the total angular momentum vector $\mathbf{J}$. Thus when an atom is placed in a magnetic field, each single state of unperturbed energy $E_{i 0}$ splits into $2 J+1$ closely spaced states having energies (in cgs Gaussian units)

$$
E_{i}=E_{i 0}+g_{i}(e \hbar / 2 m c) B m_{J}
$$

where $e, m, c$ and $\hbar$ are the electron charge and mass, the speed of light, and Planck's constant divided by $2 \pi, B$ is the magnetic field strength, $m_{J}$ is the magnetic quantum number (the projection of $\mathbf{J}$ on the field direction), and $g_{i}$ is the "Landé factor", usually between 0 and 3, that varies from one energy level to another. See Eisberg \& Resnick (1985) for more details

As a result, when an atom is placed in a magnetic field, a single transition betwen two energy levels $E_{i}$ and $E_{f}$ splits into a number of closely spaced spectral lines produced by all the allowed transitions $\left(m_{J i}-m_{J f}=0\right.$ or \pm 1$)$ between the "magnetic sublevels" of the two states. This is illustrated in Fig. 2 for the $\lambda 4574 \AA$ line of SiIII, whose lower level (with $J=1$ ) splits into three levels, while the upper level (with $J=0$ ) does not split at all. Transitions with $\Delta m_{J}= \pm 1$ are called $\sigma$ components, while those with $\Delta m_{J}=0$ are $\pi$ components.

Eq. (3.1) can easily be used to show that the wavelength shifts of the components produced by Zeeman splitting of a line at $\lambda_{0}$ are given by

$$
\Delta \lambda_{i j}=\frac{e \lambda_{0}^{2}}{4 \pi m c^{2}}\left(g_{i} m_{J i}-g_{j} m_{J j}\right)=4.6710^{-13} \lambda_{0}^{2} B\left(g_{i} m_{J i}-g_{j} m_{J j}\right),
$$

where in the second form of the equation, $\lambda$ is in $\AA$ units and $B$ is in Gauss. In the case of the $\lambda 4574 \AA$ line of SiIII, for which the upper level has $J=0$ and hence $m_{J j}=0$, and for which the lower level has $g_{i}=2.0$, the $\sigma$ components (due to lower levels with $m_{J i}= \pm 1$ ) are separated from the $\pi$ component (with $m_{J i}=0$ ) by $\Delta \lambda= \pm 2.0 \cdot 4.6710^{-13} \cdot 4574^{2} \cdot B$. Thus measurement of Zeeman splitting can be used to deduce magnetic field strength. 


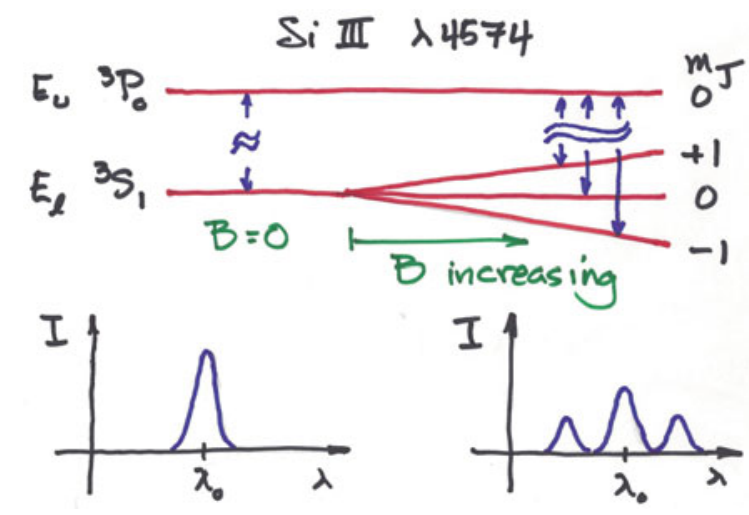

Figure 2. Splitting of the levels involved in the transition producing the $4574 \AA$ line of SirII. The upper part of the figure shows how the energy levels split as the magnetic field $B$ is increased; the two panels below sketch the appearance of this line (in emission) with $B=0$ (left) and $B \neq 0$ (right).

A further important feature of Zeeman splitting is the polarisation properties of the subcomponents of a spectral line formed in a magnetic field. Look again at Fig. 2. If the field splitting the line is parallel to the line of sight (a longitudinal field), the spectral line component (or components) arising from transitions in which $m_{J}$ changes by +1 are circularly polarised in one sense, the transitions with $\Delta m_{J}=-1$ are circularly polarised in the opposite sense, and the component with $\Delta m_{J}=0$ have zero intensity. In a transverse field, the line components formed from transitions with $\Delta m_{J}= \pm 1$ are linearly polarised normal to the field, while the components formed from $\Delta m_{J}=0$ transitions are linearly polarised parallel to the field.

These effects of a magnetic field on atomic transitions provide us with tools for measuring the strength of a magnetic field present in a star's atmosphere. This is because the splitting of spectral lines, and the polarisation properties of the various components, alter the absorption lines in the stellar spectrum. If the field is so large that the separation of $\Delta m_{J}= \pm 1$ components (called $\sigma$ components) from the central $\Delta m_{J}=0$ ( $\pi$ components) is larger than any of the other broadening mechanisms affecting the spectral line, the magnitude of the magnetic field strength $\langle B\rangle$, averaged over the visible hemisphere of the star, can be determined by using Eq 3.1. Such splitting is illustrated for the SiII multiplet (2) triplet in Fig. 3.

The polarisation produced by the Zeeman effect is also illustrated in this figure. The lower spectrum, showing the net circular polarisation spectrum (intensity of right circularly polarised light minus the intensity of left circularly polarised light, divided by total intensity, as a function of wavelength), shows a large spike of circular polarisation in each of the outer $(\sigma)$ components of the split spectral line, produced by transitions with $\Delta m_{J}= \pm 1$. Note that the net circular polarisation has opposite sign in the two $\sigma$ groups. Because circular polarisation in the $\sigma$ line components is produced only by the longitudinal component of the magnetic field, the intensity of the circular polarisation spikes allows us to estimate the strength of the line-of-sight (or longitudinal) component $\left\langle B_{z}\right\rangle$ of the magnetic field, averaged over the visible hemisphere.

However, in most cases the magnetic field is too weak, and the rotational broadening too strong, to produce clear Zeeman splitting of spectral lines. But even in stars in which the weak field does not lead to obvious splitting or at least deformation of the line intensity, the circular polarisation is often quite readily detected and measured. This is possible because the Zeeman effect effectively leads to an absorption line as seen in 


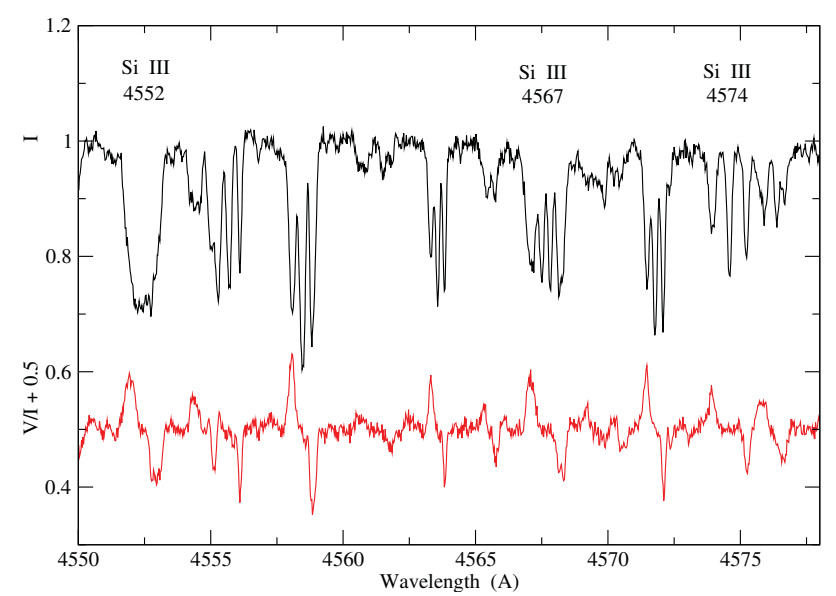

Figure 3. The upper spectrum shows Zeeman splitting in an intensity spectrum of the very strongly magnetic Bp star HD 215441. The lower spectrum shows the net circular polarisation (shifted by +0.5 for clarity). Notice that the two outer Zeeman components of each split line in the upper spectrum are strongly circularly polarised in the lower spectrum, while the central components show almost no circular polarisation.

one circular polarisation having a mean wavelength that is slightly different than the mean wavelength of the same line observed in the opposite sense of circular polarisation. Because we can measure wavelength (radial velocity) differences between spectral lines with an uncertainty that is much smaller than the width of the line, we can still detect the circular polarisation signature of a magnetic field in at much smaller strengths than those needed to produce visible splitting. This effect is illustrated in Fig. 4.

In the case illustrated in Fig. 4, the circular polarisation signature of a field is visible in each spectral line, but it is clear that if the field were ten or twenty times smaller, the signature would be lost in the noise. We can neverthless often detect such tiny polarisation signatures by noticing the obvious similarity of the polarisation signal in the different lines. This makes it possible to average both intensity and polarisation profiles of many spectral lines to bring up a detectable signal. This process is often called "least squares deconvolution", or LSD.

A more complete discussion of magnetic field meaurement using the Zeeman effect is provided by Landstreet (2009) and by Donati \& Landstreet (2009).

\section{Measuring the polarisation}

\subsection{The Stokes vector}

We now turn to the practicalities of actually measuring polarisation. We have seen that polarisation of light means that the direction of oscillation of the electric field in a beam of light has some preferential orientation and/or some net rotation. We can describe the polarisation state of the light "completely" by reporting the results of four experiments: measurements of (1) the total intensity of the beam; (2) the difference between the intensity of beam as measured through a perfect linear polariser oriented (say) vertically $\left(0^{\circ}\right)$ and one oriented at $90^{\circ}$ to the orientation of the first measurement; (3) the difference between the intensity observed when the polariser is oriented at $45^{\circ}$ and when it is at $135^{\circ}$; and (4) the difference between the intensity measured through a circular polariser that passes only right circularly polarised light and one that passes only left circularly polarised light. The last three measurements are often normalised to the total beam 


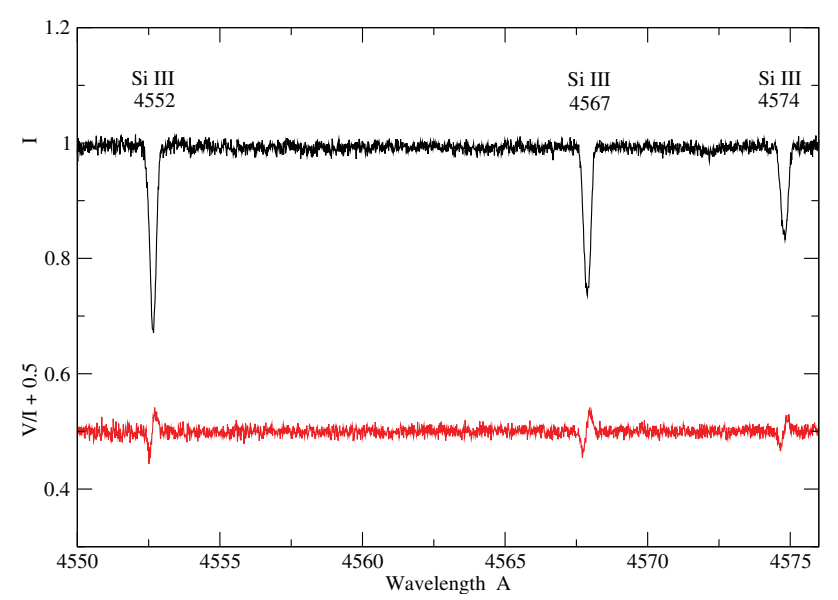

Figure 4. The upper spectrum shows an intensity spectrum of the B2p magnetic star HD 96446, with no obvious Zeeman splitting. The lower spectrum shows the net circular polarisation (shifted by +0.5 for clarity). Although no Zeeman splitting is visible in the intensity spectrum, the circular polarisation signature of a field is obvious in each spectral line.

intensity and expressed as percentages or decimal fractions. The three measurements of polarisation compare "orthogonal" polarisation state to one another.

The results of these four measurements are usually written as a vector $\{I, Q, U, V\}$, known as the Stokes vector.

The quantities in the Stokes vector usually vary with wavelength. The first component, $I(\lambda)$, is of course simply the usual intensity spectrum of a star. The other three components report how the polarisation of that light varies with wavelength. For example, a circular polarisation measurement using ESPaDOnS at the CFHT or FORS at ESO, to measure a stellar magnetic field, will result in two spectra: $I(\lambda)$ (the usual intensity spectrum) and $V(\lambda) / I(\lambda)$ (the normalised circular polarisation spectrum; see Figs 3 and 4$)$.

\subsection{How polarisation is measured}

In practice, polarisation spectra $-Q(\lambda), U(\lambda)$ or $V(\lambda)$ - are measured using normal spectrographs which have been modified by the addition of some specialised optical elements that allow one to separately meaure the intensity of the beam in two orthogonal polarisation states. These two resulting spectra are then summed to form $I(\lambda)$, and the difference provides $Q(\lambda), U(\lambda)$, or $V(\lambda)$. It would require more space than is available in this brief review to describe how these polarising optics work, and how they are actually fabricated, so I will simply describe operationally what they do. Anyone interested in more details should consult the really excellent little book "Polarized Light" by Shurcliff \& Ballard, a text from the 1960s US Commission on College Physics series.

A simple system for measuring a linear polarisation spectrum would be to place a polarizing beam splitter known as a Wollaston prism in the collimated beam of a singleorder (low-dispersion) spectrograph. A Wollaston prism splits an incident light beam into two orthogonal linearly polarised components, which leave the prism in different directions. If the beam splitter splits the beam perpendicularly to the dispersion direction, the result would be two spectra side by side. One would register the intensity $I_{0}(\lambda)$ of starlight polarised parallel to an axis at (say) $0^{\circ}$ on the sky, and the other would register the intensity $I_{90}(\lambda)$ of starlight in the orthogonal direction. From these two spectra we could obtain the $I(\lambda)=I_{0}+I_{90}$ and $Q(\lambda)=I_{0}-I_{90}$ spectra. 
Notice that we want to measure the intensities $I_{0}$ and $I_{90}$ simultaneously in order to avoid having to compare spectra taken at different times (for example with a single rotating polariser just behind the entrance slit), with different guiding, seeing and transparency conditions, and perhaps different flexure. Such sequential measurements could easily lead to difference spectra of very low accuracy.

However, the scheme I have just described is not very practical. It requires rotating the whole spectrograph by $45^{\circ}$ around the telescope axis in order to measure the Stokes $U$ component! Instead, we make use in practice of the properties of retarding wave plates to simplify the operation, increase the capabilities, and reduce the cost of our polarimeter.

The important feature of retarder wave-plates is that they allow us to convert polarisation in one of the Stokes components (say linear polarisation parallel to the $0^{\circ}$ direction) into some other polarisation form that might be easier to measure. They also allow us to exchange polarisation states so that we can have, for example, $I_{0}$ in the upper spectrum on the CCD and $I_{90}$ in the lower spectrum, and then exchange the two beams so that $I_{0}$ is in the lower CCD spectrum and $I_{90}$ in the upper. If we then compute the polarisation spectrum as the average of meaurements made in these two settings, a number of kinds of systematic errors cancel out.

It is easy to understand how wave plates affect the polarisation state of a light beam. Basically, a wave plate is a device that resolves an electromagnetic wave into two components parallel to two orthogonal axes on the face of the waveplate. As the wave travels through the wave plate, the components of the incoming wave along these two axes travel at different speeds. The phase of one component is thus shifted with respect to the other, and this changes its polarisation state.

An example can make this clear. Suppose a plane-polarised wave with its plane of polarisation at $45^{\circ}$ to each of the two wave plate axes enters the wave plate. Where the wave enters, the wave plate resolves the electric vector into components along each of the two principal axes. Since the wave is linearly polarised at $45^{\circ}$ to these axes, the two wave components oscillate in phase. If the wave plate retards one component by $1 / 4$-wave relative to the other, when the wave exits the wave plate, the maximum of the electric vector will rotate steadily in a circle from one axis to the other. The outcoming wave will now be completely circularly polarised.

If the retardation is $1 / 2$-wave, the components along the two principal axies will emerge $180^{\circ}$ out of phase. The wave will emerge linearly polarised, but aligned at $-45^{\circ}$ to the two axes instead of $+45^{\circ}$. Effectively a $1 / 2$-wave plate reflects the plane of polarisation around one of the two axes, thus rotating its plane of polarisation. This property allows us to easily exchange which of two orthogonal wave components falls onto each of the two spectra on the detector.

Thus a polarimetric analyser that can measure all the polarisation components $Q, U$ and $V$ can be constructed by using rotatable and interchangeable $1 / 4-$ and $1 / 2-$ wave plates followed by a beam-splitting polariser. This system is usually inserted into the spectrograph beam as far up the beam as possible, often before the light reaches the spectrograph entrance slit.

A much fuller discussion of the theory of polarisation measurement, and of data treatment, is provided by Bagnulo et al. (2009).

\section{Precision, accuracy, and caution}

As discussed above, polarisation is now almost always determined by computing the difference in intensity of two beams whose intensities are proportional to the two orthogonal polarisation states being measured (e.g. right and left circularly polarisation), which 
are recorded simultaneously, usually on a CCD or similar detector. Then the association of each orthogonal polarisation state with a light beam is reversed with the aid of a rotatable wave plate: if the upper CCD spectrum measured the intensity of right circularly polarised light in the first measurement, in the second it measures the intensity of left circular polarised light. By averaging the two difference spectra (after changing the sign of one), most kinds of systematic error due to different transmissivities of the two beams cancel out. The result is a measurement of one Stokes polarisation component.

If a small slit or aperture is involved (as it usually is in high resolution spectropolarimetry), seeing and guiding variations may still lead to small variations in the differences between the overall continuum level of one beam relative to the other, but the relative wavelength scales of the two beams are very well determined. Thus when the continuum polarisation is forced to zero, the difference between line profiles in the two orthogonal polarisation states, particularly any small wavelength shifts of one profile relative to the other, can be determined with much higher precision than the actual wavelength position or shape of either line profile separately. In practice, it is possible to detect and measure polarisations of substantially less than $0.01 \%$ if the data have sufficiently high signal-to-noise ratios. (Note that measuring such small polarisations requires very high photon counts per pixel, either in the original spectrum or at least in the LSD averaged spectral line.)

However, although such high precisions can be obtained with current facility spectropolarimeters, as in any precision work it is essential to be cautious about taking measured results at face value. It is extremely important to carry out polarisation observations of suitable standards such as stars showing no polarisation or known polarisation, and to compute various "check sums" which, if all is working correctly, will result in "null polarisation" spectra showing no significant signal. Only by studying the behaviour of the spectropolarimeter carefully and critically can one determine the actual precision that can be achieved.

An example of the problems that can arise from acceptance of computed precisions at face value is shown by data obtained with the FORS spectrograph at the ESO VLT. This instrument can be used as a very efficient low-resolution spectropolarimeter which can reliably detect magnetic fields of a few hundred $\mathrm{G}$ in hot stars of $m_{V} \sim 10$ or even fainter. However, Bagnulo et al. $(2012,2013)$ have shown by careful examination of the entire data set of magnetic measurements obtained with FORS1 that the actual polarimetric precision that can be achieved with this instrument is not quite as high as one would expect from the photon counts. Excess noise arises from several sources, particularly from seeing fluctuations in very short exposures, guiding variations, instrument flexures, and cosmic rays.

The presence of these extra noise sources have led to reports of numerous magnetic field detections at the $3-5 \sigma$ level in large surveys of Be stars (Hubrig et al. 2009b), of $\beta$ Cep and SPB pulsating B stars (Hubrig et al. 2009a) of O stars (Hubrig et al. 2008), and of hot subdwarfs (O'Toole et al. 2005). Reanalysis of the same data using different reduction algorithms and more conservative signal-to-noise estimates (Bagnulo et al. 2012; Landstreet et al. 2012), as well as reobservation of a number of the stars with reported fields with the ESPaDOnS spectropolarimeter at CFHT (Shultz et al. 2012), have shown that more than $80 \%$ of the magnetic field detections reported by these FORS1 surveys are spurious. The revised data show magnetic field detections in a much smaller fraction (or even none) of the observed stars than originally reported. Note, however, that with more conservative treatment and interpretation of the data, the results from these surveys are still extremely useful. 
The moral is quite simple: actually achieving extremely high precision requires extreme care and repeated verification.

To obtain the most accurate and precise magnetic field measurements of sharp line stars, there is a big advantage to using a static, stabilised high-dispersion spectrographs such as ESPaDOnS at the CFHT, NARVAL at the TBL, or HARPSpol at ESO La Silla. Such spectropolarimeters take full advantage of the precision that can be obtained from narrow line profiles, and generally lack at least some of the instabilities of Cassegrain instruments. With these instruments it is possible to confidently detect and measure magnetic fields $\left\langle B_{z}\right\rangle$ in early B stars of the order of $50-100 \mathrm{G}$ or even less if enough photons are available.

For stars with broad lines (say $v \sin i \geqslant 200 \mathrm{~km} \mathrm{~s}^{-1}$ ), low-resolution spectropolarimeters, such as ESO's FORS or ISIS at the WHT, are equally powerful choices, and the advantage brought by the large collecting area of a VLT can be very useful. For such instruments, the practical field detection limit is 200-300 G.

With today's facility instruments, it is completely practical for anyone to make spectropolarimetric observations. However, as with any unfamiliar technology, it is essential to learn about the problems and limitations of the techniques before pushing to the very highest precision limits of the instrument.

\section{References}

Bagnulo, S., Fossati, L., Kochukhov, O., \& Landstreet, J. D. 2013, A\&A 559, A103

Bagnulo, S., Landolfi, M., Landstreet, J. D., et al. 2009, PASP 121, 993

Bagnulo, S., Landstreet, J. D., Fossati, L., \& Kochukhov, O. 2012, A\&A 538, A129

Carciofi, A. C., Magalhães, A. M., Leister, N. V., Bjorkman, J. E., \& Levenhagen, R. S. 2007, ApJ (Letters) 671, L49

Davies, B., Vink, J. S., \& Oudmaijer, R. D. 2007, A\&A 469, 1045

Donati, J.-F. \& Landstreet, J. D. 2009, ARA\&A 47, 333

Eisberg, R. \& Resnick, R. 1985, Quantum Physics of Atoms, Molecules, Solids, Nuclei, and Particles, 2nd Edition

Halonen, R. J. \& Jones, C. E. 2013, ApJ 765, 17

Hubrig, S., Briquet, M., De Cat, P., et al. 2009a, Astronomische Nachrichten 330, 317

Hubrig, S., Schöller, M., Savanov, I., et al. 2009b, Astronomische Nachrichten 330, 708

Hubrig, S., Schöller, M., Schnerr, R. S., et al. 2008, A\& A 490, 793

Landstreet, J. D. 2009, in C. Neiner \& J.-P. Zahn (eds.), EAS Publications Series, Vol. 39 of EAS Publications Series, pp 1-20

Landstreet, J. D., Bagnulo, S., Fossati, L., Jordan, S., \& O’Toole, S. J. 2012, A\&A 541, A100

O’Toole, S. J., Jordan, S., Friedrich, S., \& Heber, U. 2005, A\& A 437, 227

Shultz, M., Wade, G. A., Grunhut, J., et al. 2012, ApJ 750, 2

Vink, J. S., Harries, T. J., \& Drew, J. E. 2005, A\&SA 430, 213

\section{Discussion}

NiEvA: Will the high precision be biased towards sharp-lined stars?

LANDSTREET: Yes, this is a consequence of how the measurements are made. The field of a star with many sharp and deep lines can be measured more precisely than the field of a similar star with few broad and shallow lines, just as abundances or radial velocities can be more precisely measured for the sharp-line star.

AERTS: Could you explain how to unravel line-profile shapes due to pulsations and due to a magnetic field if they act together? i.e. what is the effect of pulsation on your magnetic modelling? 
LANDSTREet: This is discussed in the talk of Coralie Neiner.

HERRERO: Instruments and telescopes introduce additional polarisation through reflections. Is it better to use standards to calibrate the observations or theoretical knowledge about the instrument+telescope behaviour?

LANDSTREET: One should use all available means to understand instrumental polarisation and phase shifts. However, most polarimetric analysers today are at Cassegrain focus, where the axial symmetry and nearly normal reflections from primary and secondary mirrors introduce almost no instrumental polarisation or phase shifts. After the polarisation analyser optics, the polarisation measurement becomes a comparison of two intensities, and any polarising properties of the optical train are unimportant.

PULS: Could you comment on potential depolarisation effects by the ISM (inhomogeneities, B-fields, etc) for the stars we are interested in?

LANDSTREET: This is an interesting possibility, but I do not know of any work that has considered it.

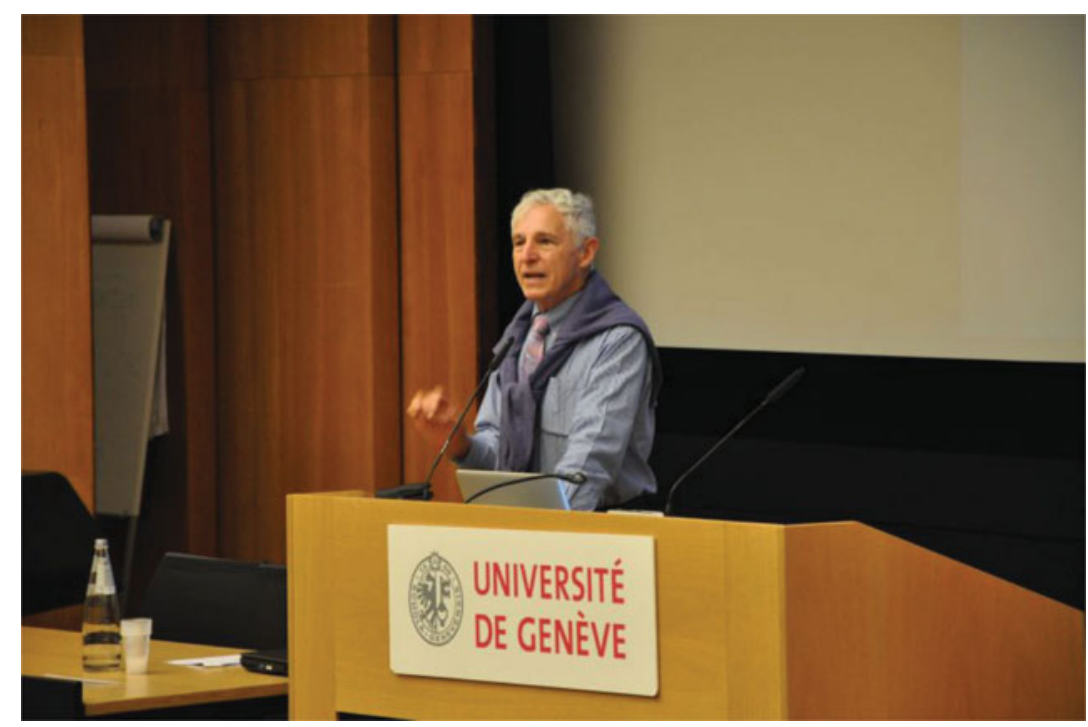

John Landstreet 